\title{
Biophysical
} Chemistry

\section{Recent results on the connection between thermodynamics and dynamics in supercooled water}

\author{
Francis W. Starr a* , C. A usten A ngłll, Emilia La Nave, Srikanth Sastify, A ntonio Scâla, \\ Francesco Sciortino ${ }^{\mathrm{c}}, \mathrm{H}$. Eugene Stanley ${ }^{\mathrm{e}}$ \\ ${ }^{a}$ Polymers Division and Center for Theoretical and Computational Materials Science, \\ National Institute of Standards and Technology, 100 Bureau Dr., MS 8500, Gaithersbury, MD 20899, USA \\ ${ }^{\mathrm{b}}$ Department of Chemistry, Arizona State University, Tempe, AZ 85287, USA \\ ${ }^{c}$ Dipartmento di Fisica e Istituto Nazionale per la Fisica della Materia, Universitá di Roma 'La Sapienza', Piazzale Aldo Moro 2, \\ I-00185, Roma, Italy \\ dJawaharlal Nehru Center for Advanced Scientific Research, Jakkur Campus, Bangalore 560064, India \\ ${ }^{\mathrm{e}}$ Center for Polymer Studies, and Department of Physics, Boston University, Boston, MA 02215, USA
}

Received 3 January 2003; received in revised form 31 January 2003; accepted 31 January 2003

\begin{abstract}
We review recent results on the connection between thermodynamics and dynamics in a model for water. We verify the A dam-Gibbs relation between entropy and dynamic properties using computer simulations, which allow direct access to the relevant properties. We combine experimental measurements of entropy with the A dam-Gibbs hypothesis to predict dynamic properties in deeply supercooled states, which are difficult to access experimentally. We find evidence suggesting that the glass transition temperature of water may be significantly higher than previously reported, but is still consistent with recent measurements. Fnally, we discuss the hypothesis that the dynamical behavior of deeply supercooled water undergoes a crossover from 'fragile' to 'strong' behavior.
\end{abstract}

(c) 2003 Elsevier Science B.V. A 11 rights reserved.

Keywords: Fragile; Experimentally; Hypothesis; Thermodynamics

\section{Introduction}

The seminal book of Professor Kauzmann taught everyone that water is widely studied for many practical reasons-it is the most ubiquitous fluid

\footnotetext{
D edicated to Professor W. Kauzmann, whose seminal 1969 book has inspired much of this research.

* Corresponding author. Tel..+1-301-975-8359; fax: +1301-975-5012.

E-mail addresses: fstarr@nist.gov (FW. Starr ), hes@bu.edu (H.E. Stanley).
}

on earth, and its unusual properties are thought to be related to basic phenomena in biology, chemistry, and engineering [1-5]. Frequently, non-specialists regard these unusual properties as unique to water. However, recent simulation studies have suggested that the temperature dependence of many dynamic properties are not altogether unlike those of some simple liquids [6-9]. For example, supercooled liquid water seems to be one among many liquids that can be described over a range 
of temperatures by the mode-coupling theory ( $\mathrm{TT}$ ) [10]. NT provides quantitative predictions for the temperature dependence of relaxation times for the dynamics of supercooled liquids in the 'weakly' supercooled regime, where $D$ decreases by approximately three orders of magnitude from the typical liquid value. However, as temperature decreases toward a 'dynamical critical temperature' $T_{\mathrm{MT}}$ predicted by NT theory, the NT breaks down. Typically, $T_{\mathrm{NaT}}$ is $\approx 50 \%$ larger than the experimentally measured $T_{\mathrm{g}}$, and relaxation times are still approximately 10 orders of magnitude smaller at $T_{\text {NTT }}$ than at $T$. Hence there is a huge range of $T<T_{\mathrm{NCT}}$, the 'deeply' supercooled region, where dynamic properties change dramatically, but the underlying reasons for this behavior are not understood. A dditionally, glass-forming liquids are typically categorized as (i) strong liquids - those having an A rrhenius $T$ dependence of dynamic properties with an activation energy of roughly $37 R T$ and (ii) fragile liquids - those having a strongly non-A rrhenius $T$ dependence of dynamics properties [11-13]. Water behaves as a fragile liquid in the experimentally accessible region where $\mathrm{T}$ predictions seem to apply. In the range $T<T_{\text {NGT }}$, there have been suggestions that water may behave quite differently than other liquids.

Unfortunately, simulations of equilibrium properties for $T<\mathrm{T}_{\text {naT }}$ are not feasible at the present time due to the excessively large relaxation time, relative to the computational time required. A dditionally, experiments on liquid water are hampered by the fact that even carefully prepared samples of supercooled water crystallize at the homogeneous nucleation temperature' $T_{\mathrm{H}}=235 \mathrm{~K}[3,14$, slightly above the expected value of $T_{\mathrm{N} T \mathrm{~T}}$ for water [15]. O the other hand, liquid water can be vitrified at atmospheric pressure by a variety of methods, including quenching aerosol droplet samples to liquid nitrogen temperatures [16]. Glassy water crystallizes on heating at $T_{X} \approx 150 \mathrm{~K}$ making the properties of amorphous water in the range $T_{X}<T<T_{\mathrm{H}}$ almost unknown. While the properties of glassy water, referred to as low-density amorphous ice (LD A), are intrinsically non-equilibrium, a careful analysis of the thermodynamic properties of glassy water and their relation to the properties of equilibrium samples at $T>T_{\mathrm{H}}$ can possibly help provide insight into the expected properties in the difficult- to- probe region $T_{X}<T<$ $T_{\mathrm{H}}$.

A $n$ evaluation of the thermodynamic data in this range is particularly valuable, owing to the development of a formalism relating thermodynamics to dynamics in deeply supercooled states. These ideas reach back to the seminal work of Kauzmann [17], and have been expanded over time by the work of, among others, A dam and Gibb\$18], Goldstein [19], and Stillinger and Weber [2 0, \. 1 The central idea underlying the approach is that dynamics at low temperatures are controlled primarily by the statistical properties of the underlying potential energy landscape [2 $\$$; this landscape consists of a tremendous number of local minima corresponding to mechanically stable amorphous states. A t sufficiently low $T$, the system is expected to be localized within one of these basins, with infrequent 'activated processes', which bring the system to a new basin. Several authors have studied the landscape properties explicitly and it appears that, at $T_{\mathrm{NTT}}$ (where the NT predictions break down), the system motion becomes dominated by inter- basin 'hopping' via regions of collective motion [2 3-37. Hence the statistical properties of the landscape is key to extending the quantitative understanding of supercooled liquids to the region $T<T_{\text {nat }}$.

In this testimonial to Professor Kauzmann, we examine the implications of both experimental and computational studies for the dynamic properties of liquid water in the deeply supercooled region, where little equilibrium data are available. From simulations, we establish the validity of the A dam-Gibbs relationship between entropy and dynamic properties, like the diffusion constant $D$ [35-38]. Having validated this approach, we consider experimental measurements of the entropy for the liquid for $T>T_{\mathrm{H}}$ and glassy water at $T<$ $T_{X}$. By applying simple, but somewhat tedious constraints of thermodynamics, we can predict the expected behavior of the entropy in the range $T_{X}<$ $T<T_{\mathrm{H}}$. The results for the entropy, when combined with the A dam-Gibbs relation, suggest that water should crossover from fragile liquid behavior to strong liquid behavior at $T<T_{\mathrm{H}}$ [39], as first 
proposed in Ref. [40 ]. If these predictions are valid, water again appears as an anomalous liquid, and deserves careful scrutiny. A dditionally, results reported in Ref. [39] suggest that $T_{\mathrm{g}} \approx 160 \mathrm{~K}$ is far above traditionally accepted estimates of $T_{\mathrm{g}}$, but consistent with recent results based on hyperquenching [41].

\section{Background}

In 1948, Kauzmann published a seminal paper that focused attention on the interplay of thermodynamics and dynamics of a liquid near its vitrification temperature [17]. We briefly review the theoretical foundations of the thermodynamic viewpoint of the glass transition that grew from his work. This is an incomplete account; a more complete account can be found in Ref. [2].

Kauzmann focused on the entropy of the liquid and its corresponding crystal. O cooling, the liquid entropy decreases at a much faster rate than the crystal entropy. Hence, by naive extrapolation, we expect $S_{\text {liquid }}=S_{\text {crystal }}$ at a temperature $T_{\mathrm{K}}$, the Kauzmann temperature, and $S_{\text {liquid }}<S_{\text {crystal }}$ for $T<$ $T_{\mathrm{K}}$. While it is strange to imagine a liquid with a smaller entropy than the crystal, it is not thermodynamically inconsistent. The situation is more problematic considering that, for most systems, $\mathrm{S}_{\text {crystal }}(T \rightarrow 0)=0$, which would require that $S_{\text {liquid }}<0$, which is inconsistent with the classical expression $S=k_{\mathrm{B}} \ln \Omega$ since the number of states $\Omega$ cannot be less than unity.

The 'Kauzmann paradox' refers to the fact that, in practice, the kinetic glass transition intervenes so as to avoid the thermodynamic "entropy catastrophe'-dynamics saving thermodynamics. To avoid the possibility of an entropy catastrophe without a paradox, Kauzmann proposed that the barrier to crystallization vanishes at some $T$ between $T_{\mathrm{K}}$ and $T_{\mathrm{g}}$, thereby avoiding the equal entropy point by simple crystallization. Subsequent work considered the possibility that crystallization might not intervene, and put forth the notion that $T_{\mathrm{K}}$ may be the point of an 'ideal' thermodynamic glass transition, attainable only by an infinitely slow cooling of the liquid [Q⿻ $]$ ]. In practice, such a transition is never observed, as laboratory measurements of $T_{\mathrm{g}}$ depend on the cooling rate.
The notion of a thermodynamic singularity underlying the glass transition was expanded on by the work of A dam, Gibbs' and D ildrzio. They developed a theoretical framework in which the liquid at low $T$ evolves through cooperatively rearranging regions, rather than simple Brownian motion as at high $T$, and associated the size of the cooperatively rearranging regions with a configurational entropy $S_{\text {conf. }}$. Mst importantly, since the rearranging region controls the relaxation of the liquid, they proposed that the relaxation time $\tau$ (or other dynamic properties, like the diffusion constant $D$ ) are related to $S_{\text {conf }}$ via

$\ln \tau \sim\left(T S_{\text {conf }}\right)^{-1}$.

In this scenario, an ideal glass forms when the relaxation time diverges, and hence $S_{\text {conf }}=0$. Thus, it has become common to refer to $T_{\mathrm{K}}$ also as the $T$ where $S_{\text {conf }}$ vanishes. The concept of cooperative rearrangement and a close relation of the dynamics to the configurational entropy have remained central to the development of the theoretical approach.

The primary remaining question is: what is $S_{\text {conf }}$ and how can it be measured? The first steps to resolving this question were made by Goldstein [19], who focused on the underlying potential energy landscape, arguing that the dynamics of a liquid can be split into two contributions at low $T$ : (i) vibrations localized within a single basin of a landscape, and(ii) infrequent hops between basins that give rise to structural relaxation. In this context, there is a natural separation of $S$ into a vibrational contribution $S_{\text {vib }}$ and a configurational contribution $S_{\text {conf, }}$ arising from the number of basins the liquid samples. From the point of view of the energy landscape, the ideal glass is formed when the liquid becomes trapped in a single landscape basin.

Stillinger and Weber formalized the concept of a basin in the energy landscape by introducing the inherent structure (IS) formalism. Specifically, the set of points that map to the same minimum, or IS, are those which constitute a basin. This approach is particularly well suited to simulated liquids, since it is possible to explicitly calculate the steepest descent to a local minimum from an equilibrium configuration $[\mathcal{B}]$. Mreover, the par- 
tition function can be explicitly rewritten in terms of the basins. By introducing the density of states $\Omega\left(E_{\mathrm{IS}}\right)$, i. e. , the number of minima with energy in the interval of energy $E_{\mathrm{IS}}$ to $E_{\mathrm{IS}}+d E_{\mathrm{IS}}$ we can rigorously define

$S_{\text {conf }}=k_{\mathrm{B}} \ln \Omega\left(E_{\mathrm{IS}}\right)$.

The Helmholtz free energy is given by

$F=E_{\mathrm{IS}}-T S_{\text {conf }}+f_{\text {basin }}\left(T, E_{\mathrm{IS}}\right)$.

Here $f_{\text {basin }}$ captures the vibrational contribution and kinetic degrees of freedom. E q(3) is a formal expression for the separation of configurational and vibrational contributions. For our purposes, we will focus on the fact that one can define $S_{\text {conf }}=S-S_{\text {vib }}$, and evaluate $S$ and $S_{\text {vib }}$ to obtain $S_{\text {conf }}$.

\section{Calculation of configurational entropy of simulated water}

We calculate the absolute entropy at all state points simulated by following the procedure described in Ref. [4 ]. We use the ideal gas as a reference state and use thermodynamic integration to link to a system at high $T$ and small $\rho$ (large volume $V$ ), where interactions are far less important. The entropy at all other $(\rho, T)$ simulated are then obtained by a numerical integration of the relationship $d S=\frac{1}{T} d E-\frac{p}{T} d V$. The only additional complication beyond previous calculations for Lennard-Jones systems is that we must consider the reference state to be a tri-atomic ideal gas, rather than a monatomic one. The tri-atomic ideal gas entropy is given by

$$
\begin{aligned}
S_{i d}(V, T)= & N k_{\mathrm{B}}\left[\ln \frac{\pi^{1 / 2} V}{N h^{6}}+\frac{3}{2} \ln \left(2 \pi m k_{\mathrm{B}} T\right)\right. \\
& \left.+\frac{1}{2} \sum_{i=1}^{3} \ln \left(8 \pi^{2} I_{i} k_{\mathrm{B}} T\right)+4-\ln 2\right],
\end{aligned}
$$

where $I_{i}$ are the moments of inertia, and $h$ is Planck's constant.
Qr primary goal is to calculate $S_{\text {conf }}$ so that we may test proposed relations between the dynamic properties and thermodynamic properties. We exploit the hypothesized relationship that $S=$ $S_{\text {conf }}+S_{\text {vib. }}$. For $T$ not far from the mode-coupling temperature $T_{\mathrm{NTT}}$, Ref. [4] observed that the vibrational contribution may be well approximated by a classical harmonic solid, with the eigenfrequencies defined by the inherent structures sampled below the 'onset temperature' $[\mathcal{B}, 45,46]$. Unfortunately, even at the lowest $T$ studied, the extended simple point charge (SPC/E) model of water displays a significant anharmonic contribution to $U$. To approximate the contribution due to the anharmonicity of the potential, we include higher order terms in an expansion of the vibrational energy

$U_{\mathrm{vib}}=3 k_{\mathrm{B}} T+a T^{2}+b T^{3}$

Here $a$ and $b$ are fitting parameters, and the harmonic contribution to the energy is given by $3 k_{\mathrm{B}} T$. Since $(d U / d T)_{v}=T(d S / d T)_{v}$, we may express the vibrational entropy as

$S_{\text {vib }}=S_{\text {harmonic }}+S_{\text {anharmonic }}$

$=\frac{k_{\mathrm{B}}}{N} \sum_{i=1}^{6 N-3}\left[\ln \left(\frac{k_{\mathrm{B}} T}{\hbar \omega_{i}}\right)+1\right]+2 a T+\frac{3}{2} b T^{2}$,

where $\left\{\omega_{i}\right\}$ are the eigenvalues of the Hessian matrix $\partial^{2} V / \partial x_{j} \partial x_{k}$. We calculate $\left\{\omega_{i}\right\}$ from the normal mode spectrum of the liquid after quenching to the inherent structure at each $(\rho, T)$ system point simulated.

We only need to evaluate the constants $a$ and $b$ to obtain $S_{\text {vib }}$. We heat the quenched structures and measure the dependence of $U$ on $T$, which allows us to fit $U$ to the form specified in Eq. (5). In order to obtain reliable fits of the constants, the heating schedule follows the following sequence:

- The temperature is first increased to $50 \mathrm{~K}$ for 10 ps to anneal the effect of any nearby minima that may be lower in energy due to the extreme roughness of the landscape.

- The temperature is reduced to $1 \mathrm{~K}$ for $10 \mathrm{ps}$, to allow the vibrational degrees of freedom to 


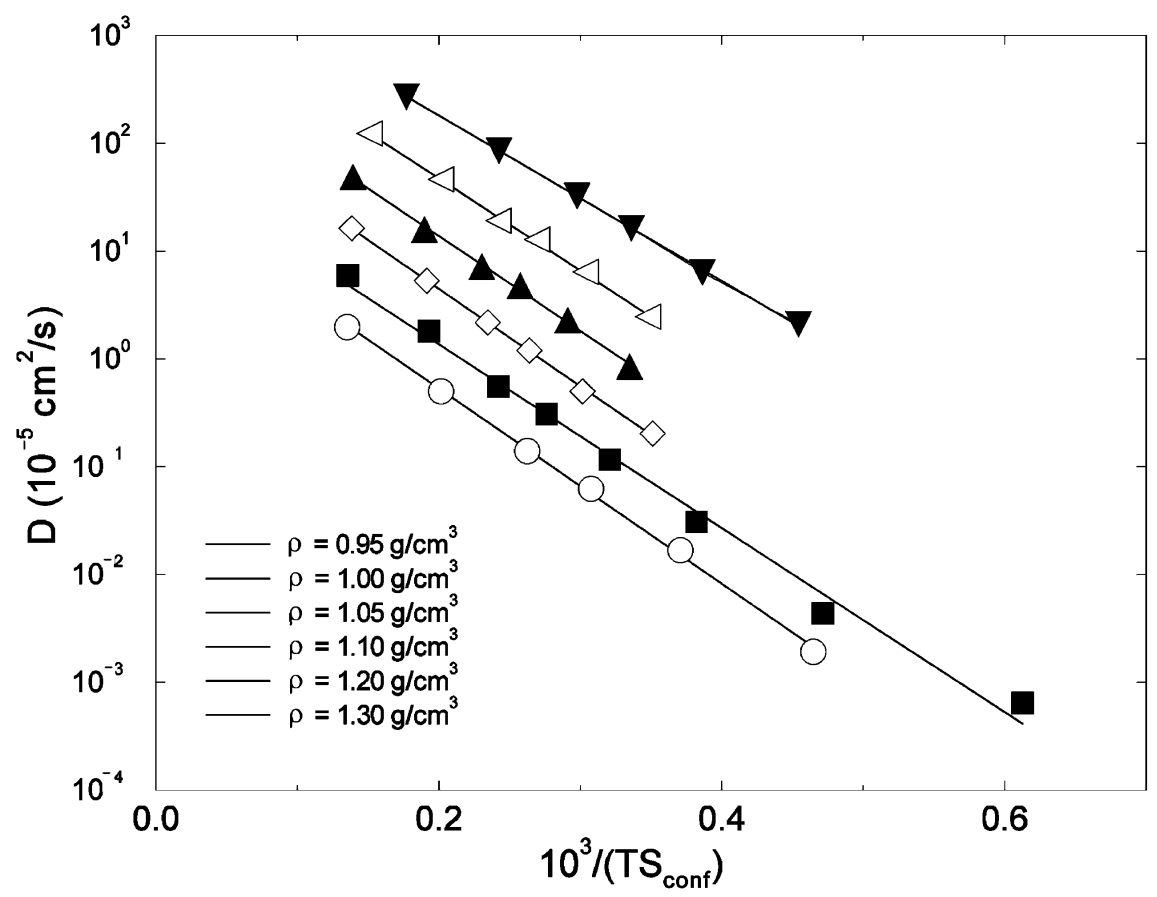

Fig. 1. The diffusion constant $D$ as a function of $1 / T S_{\text {conf }}$ for the SPC/E model, confirming the predicted relation of A dam and Gibbs. Note that the curves for each density have been shifted for clarity. Figure redrawn from Ref.[35]. Verification of the proposed relation for simpler models is reported in Refs. [ $46-49]$.

equilibrate, followed by 10 ps of data collection in the NVE ensemble.

- The temperature is then increased to $10 \mathrm{~K}$ and then by $10 \mathrm{~K}$ intervals following the same schedule of 10 ps for the equilibration of vibrational degrees of freedom and 10 ps of data collection in the NVE ensemble up to $190 \mathrm{~K}$ at higher $T$, diffusive motion becomes significant on these time scales.

A t this point, we have both the total and vibrational entropy for all state points simulated and can now calculate $S_{\text {conf }}=S-S_{\text {vib }}$.

We also briefly discuss the calculation of the entropy of crystalline forms, since we want to compare the properties of $S_{\text {conf }}$ and the excess entropy $S_{\text {ex }}=S_{\text {liquid }}-S_{\text {crystal. }}$. From the landscape point of view, a crystalline arrangement corresponds to only a single basin, since significant rearrangement of the molecules would destroy the crystalline structure. Hence, one expects the crystal entropy to be entirely vibrational in nature. Thus, we calculate $S_{\text {crystal }}$ using the same techniques used to calculate $S_{\mathrm{vib}}$ of the liquid inherent structures. However, since the crystal structure does not change (i. e. the system is always exploring the same crystalline basin), the normal modes $\left\{\omega_{i}\right\}$ are independent of $T$, but of course still depend on $\rho$. Quenches from several $T$ confirm this.

\section{Testing the Adam-Gibbs hypothesis}

Having obtained $S_{\text {conf }}$ over a wide range of density at supercooled temperatures, we can test the proposed relation

$\ln D \sim\left(A / T S_{\text {conf }}\right)$.

In the range of $D$ values where bulk water experiments have been performed, we find agreement with the proposed relationship for the SPC/ E model of water, as shown in Fig. 1.

E valuation of $S_{\text {conf }}$ relies on the ability to perform instantaneous quenches that follow a steepest descent path (or alternatively, a conjugate gradient minimization). Since this approach is not experi- 


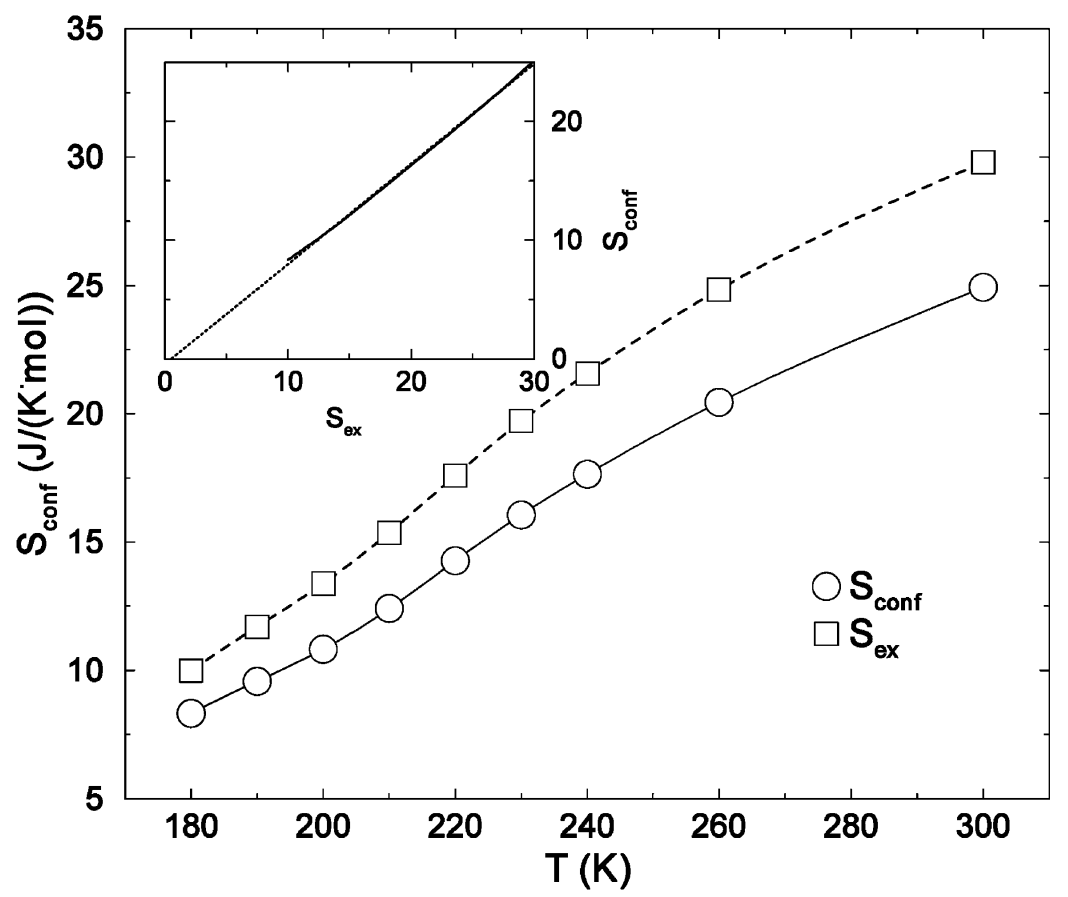

Fig. $2 S_{\text {conf }}$ and $S_{\text {ex }}$ at a fixed density $\rho=1.0 \mathrm{gcm}^{3}$ for the SPC/E model. The inset shows a parametric plot(solid line) to demonstrate proportionality. Figure redrawn from Ref. [39].

mentally feasible, many experimentalists have exploited the fact that crystalline entropy is vibrational in nature and have approximated $S_{\mathrm{vib}}=$ $S_{\text {crystal, }}$ and hence $S_{\text {conf }} \approx S_{\text {ex }}$. This approximation has been found to work well in the approach of A dam and Gibbs. However, we do not in general expect that $S_{\mathrm{ex}}=S_{\text {conf }}$, since it would imply that the liquid and crystalline basins have the same eigenfrequencies (or, loosely speaking, shapł and that the liquid frequencies are invariant $[36,5]$ Indeed, we see that $S_{\text {ex }}$ and $S_{\text {conf }}$ differ for the SPC /E model (Fig. 2). D etails of the ice simulation are given in Ref. [36]. Hence the successful use of $S_{\text {ex }}$ can only be explained if $S_{\text {ex }} \propto S_{\text {conf }}$, as pointed out in Ref. [51,5]; in such a case, the constant of proportionality can be absorbed into the free parameter of the A dam-Gibbs equation. To test this possibility, we have made a parametric plot of $S_{\text {conf }}$ and $S_{\mathrm{ex}}$ in the inset of Fig. 2, demonstrating the linear proportionality and hence explaining why $S_{\text {ex }}$ can be substituted for $S_{\text {conf }}$.

\section{Application of the Adam-Gibbs hypothesis to experimental data}

\subsection{Estimation of entropy for $T<T_{H}$}

To determine a reasonable form for the entropy $S=S(T, P)$ in the range $T_{X}<T<T_{\mathrm{H}}$, we first focus on thermodynamic properties that facilitate the calculation of $S$ in the easily-accessible regions $T>T_{\mathrm{H}}$ and $T<T_{X}$, and whose values also place strict limits on the possible behavior of $S$ in the region $T_{X}<T<T_{\mathrm{H}}$. Like $S_{\mathrm{ex}}$, all other 'excess' quantities, such as specific heat $C_{P}^{\mathrm{ex}}$ and enthalpy $H_{\text {ex }}$, refer to the difference between liquid and crystalline states. E ach of these three quantities is known experimentally for $T>T_{\mathrm{H}}$ as well as $T<$ $T_{X}$.

In the difficult-to- probe $T_{X}<T<T_{\mathrm{H}}$ region, we construct two possible forms for $S_{\mathrm{ex}}$. To connect the regions $T>T_{\mathrm{H}}$ and $T<T_{X}$, we must consider the thermodynamic constraints on the entropy. These constraints are: 


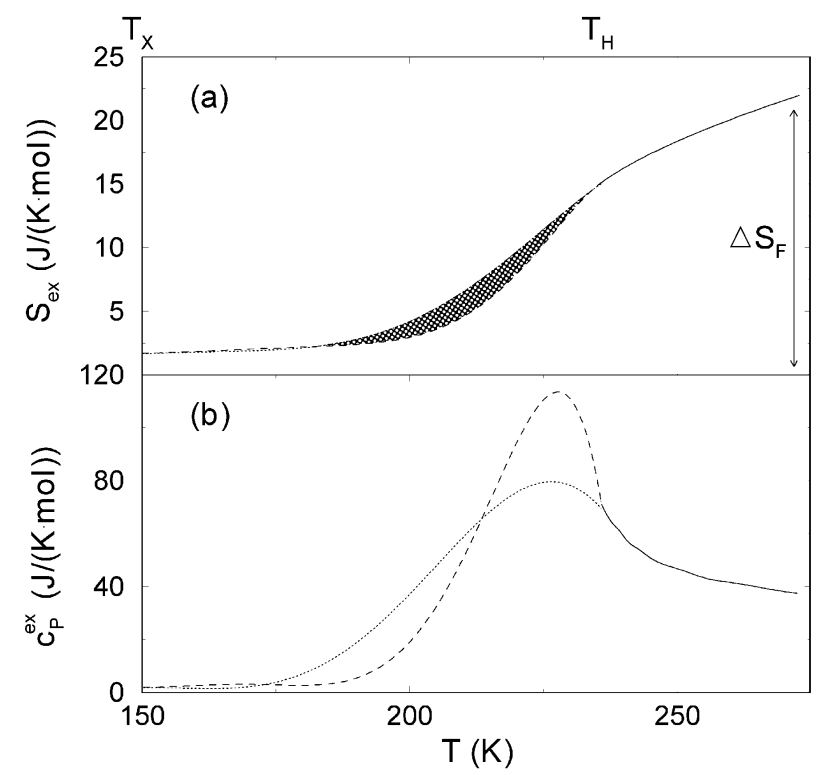

Fig. 3. (a) Possible forms for the excess entropy $S_{\mathrm{ex}}$ in the experimentally inaccessible region. The two curves show the fits obtained using the upper and lower bounds on the area under $S_{\text {ex }}$. The entropy of fusion $\Delta S_{\mathrm{F}}=21.8 /(\mathrm{K}$ mol ) for freezing at $273 \mathrm{Kis}$ indicated by the arrow, (b) Constant pressure excess specific heat $C_{P}^{\mathrm{ex}}=T\left(d S_{\mathrm{ex}} / d T\right) p$ for the possible forms of $S_{\text {ex }}$ shown in (a). Figure redrawn from Ref. [39].

(I-IV) $S_{\text {ex }}$ and $C_{P}^{\text {ex }}$ at the endpoints and the slopes of $\mathrm{S}_{\mathrm{ex}}$ at $T_{X}$ and $T_{\mathrm{H}}$-four constraints.

(V) $S(T)$ must be a monotonic increasing function because $C_{P}^{\mathrm{ex}} \geq 0$. Note that this is not a uniquely defined constraint.

(VI) The area $A$ under the curve $S(T)$ is defined by the excess Gibbs free energy $G_{\mathrm{ex}}$.

$A=\left[T S_{\mathrm{ex}}-H_{\mathrm{ex}}\right]_{T_{X}}^{T_{\mathrm{H}}}$.

Or challenge is to determine a functional form for $S_{\mathrm{ex}}(T)$, given only its values at the limiting temperatures $T_{X}$ and $T_{\mathrm{H}}$, the area $A$ under $S(T)$, and the monotonicity of $S(T)$. Related work on the possibility of a liquid-liquid phase transition [53-69 suggests that $S(T)$ should not contain a discontinuity at atmospheric pressure, and hence we aim to develop a form for $S(T)$ (and its derivatives) that varies continuously [70 -72].

We show possible forms of $S_{\text {ex }}$ that satisfy the upper and lower bounds on the area constraint of $S_{\text {ex }}$ in Fig. 3. These two curves represent approxi- mate bounds on the form of $S_{\text {ex }}$ in the unknown region; these bounds are somewhat larger if the uncertainty in $S_{\mathrm{ex}}$ is also included. Fig. 3 shows that $S_{\mathrm{ex}}$ and $C_{P}^{\mathrm{ex}}$ both display these significant changes in their behavior below $230 \mathrm{~K}$ This is a result of the fact that $S_{\mathrm{ex}}$ must remain nearly constant near $T_{X}$ in order to satisfy the constraint of Eq. (9). The inflection in $S_{\text {ex }}$ (Fg. 3) must occur at $T \gtrsim 215 \mathrm{~K}$ were the inflection to occur at a significantly lower temperature, the area $A$ bounded by $S(T)$ would be too large.

\subsection{Prediction of dynamic properties}

Having established the legitimacy of the A damGibbs equation, as well as the proportionality between $S_{\text {conf }}$ and $S_{\text {ex }}$, we now consider applying the A dam-Gibbs equation to our estimated values for $S_{\text {ex }}$ in the deeply supercooled region $T<T_{H}$.

We select proportionality constants in the A dam-Gibbs equation to fit $S_{\text {ex }}$ to $\eta[73,74]$ and $D$ [75] (Fg. 4) for $T>235 \mathrm{~K}$ where experimental measures of all quantities are available. The superA rrhenius behavior for $T \geq 230 \mathrm{~K}$ is typical for a fragile liquid $[12,1] 3$ The maximum in $\mathbb{C}_{P}^{x}$ (approximately 225 )Kis reflected by the inflection of $\eta$ and $D$; this change is not clearly evident in $\eta$ or $D$ until $T \lesssim 190$, where the dynamic properties are approximately A rrhenius. In contrast to the fragile behavior for $T$ close to $T_{\mathrm{H}}$, the behavior for $T$ near $T_{X}$ is characteristic of a strong liquid $[12,1] 3$ which is A rrhenius behavior with an appropriate activation energy. Here we find an activation energy $E \approx 74 \mathrm{~kJ} / \mathrm{mol}$, which converts to a 'fragility index' $m=E / 2.30 R 3 T_{\mathrm{g}}=28$, if we use $T_{\mathrm{g}}=136 \mathrm{~K}$ or $m=24$ if we use $T_{\mathrm{g}}=160 \mathrm{~K}$ comparable to $m$ for sodium trisilicate (a very strong liquid) [77]. Such a crossover from fragileto-strong behavior is not typical of liquids [78], but does appear in simulations of $\mathrm{BeF}_{2}$ and $\mathrm{SiO}{ }_{2}$ $[79,8 \Phi$, which, like water, have a tetrahedral network structure [8 1]. The value of $E$ is consistent with that obtained experimentally from crystalli-

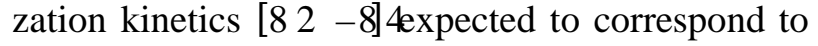
the activation energy of the diffusion constant of the crystallizing phase [85]. However, we point out that these crystallization kinetics-based results 


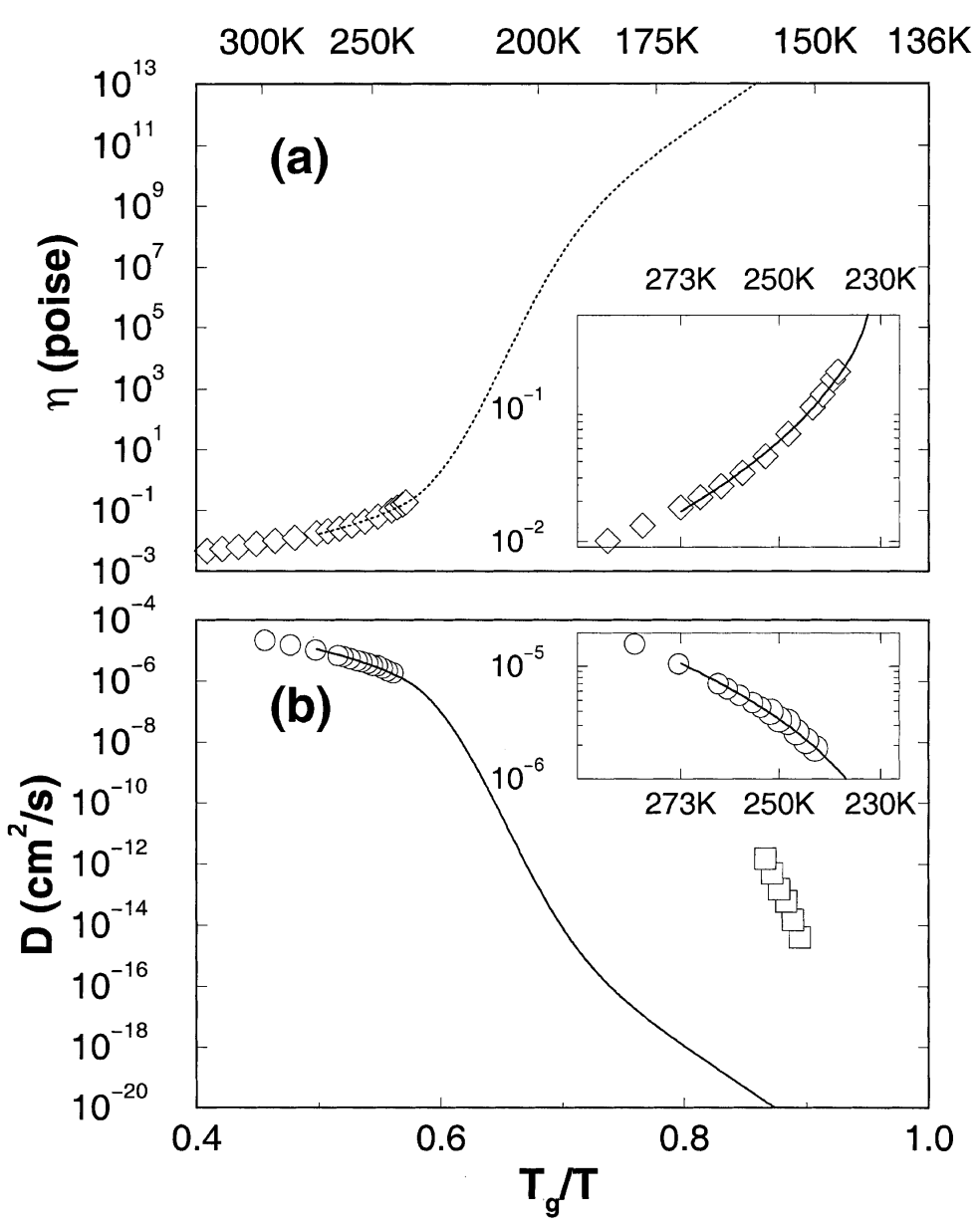

Fig. 4 (a) Fit of $S_{\text {ex }}$ and viscosity $\eta$. Experimental data $(\diamond)$ are from [73, 74]. D iffusion constant $D$ predicted using the same method. The experimental data $(\bigcirc)$ for $T>235 \mathrm{~K}$ are from[75]. The data for $T<160 \mathrm{~K}(\square)$ are from [76]. (b) Both (a) and (b) show behavior expected for a strong liquid for $T \leqq 220 \mathrm{~K}-\mathrm{i}$. e. A rrhenius behavior with an activation energ $\bar{F}_{\mathrm{g}} / 3$ (in units of $\mathrm{kJ} / \mathrm{mol}$ ) [12]. The insets show the quality of the fit in the region where experimental data are available. Figure redrawn from Ref. [39].

are in conflict with the evaporation-rate based diffusivity results of Ref. [76], which obtain $E \approx 170 \mathrm{~kJ} / \mathrm{mol}$, indicating that further tests are necessary to determine the properties of water in this region.

For most systems, the value of $\eta\left(T_{\mathrm{g}}\right) \approx 10^{13}$ Poise, while Fig. 4 shows that $\eta$ reaches this value at $T \approx 160 \mathrm{~K}$ significantly higher than the expected $T_{\mathrm{g}}=136 \mathrm{~K}$ This may be an indication of the limitations of our approach for estimating dynamic properties. A lternatively, this may be an indication that $T_{\mathrm{g}}$ of water is in fact significantly higher than $136 \mathrm{~K}$ Velikevet al. [4l] shows that the thermal data for hyper- quenched glassy water are incompatible with what is known about the relaxation of trapped enthalpy from other hyperquenched glasses, and that the incompatibility can only be resolved if the data for water are re-scaled using a glass transition temperature of 165-170 $\mathrm{K}$ This roughly coincides with the $T_{\mathrm{g}}$ predicted by Fig. 4 While a simple linear extrapolation of binary aqueous solution data suggests $T_{\mathrm{g}}=136 \mathrm{~K}$ for pure water, linear extrapolations are unreliable if the target substance is a network forming liquid, like water [8 6. A n alternate extrapolation, applicable to other network forming liquids, suggests 
that $T_{\mathrm{g}}$ for water is considerably higher than 136 $\mathrm{K}[8 \mathrm{6}$. However, this remains a matter of controversy $[87]$.

If the assignment of $T_{\mathrm{g}} \approx 160 \mathrm{~K}$ is correct, then the data used at $T=150$ Krefers to a glassy state, and hence $S_{\text {ex }}(150$ 內 would be smaller for an equilibrium state. Fortunately, even if the data at $150 \mathrm{~K}$ are out of equilibrium, it does not severely effect our estimates because the value of $S_{\text {ex }}(150$ $\mathrm{K})$ is already extremely small, and further equilibration at the $T$ would only reduce $S_{\text {ex }}$ closer to zero. This would result in a slightly more pronounced inflection on $S_{\mathrm{ex}}$ than we have anticipated here.

\section{Conclusion}

We have presented evidence from simulations supporting the use of A dam-Gibbs equation to interpret the dynamics of supercooled liquids. In the case of liquid water, experimental data, when combined with the A dam-Gibbs equation, suggest that water undergoes an unusual fragile- to-strong crossover, which may prove important in technological uses of vitreous water, such as biopreservation. A dditionally, we have reviewed results supporting the conjecture that $T_{\mathrm{g}}$ may be significantly higher than previously expected.

\section{Acknowledgments}

We thank S. V. Buldyrev, P. G. D ebenedetti, J. F D ouglas, G. Franzese, N. Giovambattista, S. C. Glotzer, M Mlnques, R. Sadr and M Yamada for helpful discussions, and NSF Chemistry Grant CHE - 0096892 for support.

\section{References}

[1] D. E isenberg, W. Kauzmann, The structure and properties of water, Qford University Press, New York, 1969.

[2] P. G. D ebenedetti, Mtastable liquids, Princeton University, Press, Princeton, 1996.

[3] C. A. A ngell, Supercooled Water, in: F Fran(lks d), Water: A Comprehensive Treatise, 7, Plenum Press, 1982 , pp. 2 15-338.

[4] M- C. , Bellissent- Funel. Hydration processes in biology: theoretical and experimental approaches [Proceedings NA TOA dvanced Study Institutes, vol. 30 5\& Press, A msterdam, 1999.
[5] V. Brazhkin, S. V. Buldyrev, V. N. Ryzhov, H. E. Stanley, eds. , New kinds of phase transitions: transformations in disordered substances [Proc. NA TOA dvanced Research Workshop, Volga Rivel Kuwer, D ordrecht, 2002 .

[6] P. Gallo, F Sciortino, P. Tartaglia, S. - H. Chen, Slow dynamics of water molecules in supercooled states, Phys. Rev. Lett. $7(6996) 2730-2733$.

[7] F Sciortino, P. Gallo, P. Tartaglia, S. - H. Chen, Supercooled water and the kinetic glass transition, Phys. Rev. Part E 54(1996) 6331-6 346.

[8] F W. Starr, S. Harrington, F Sciortino, H. E. Stanley, Slow dynamics of water under pressure, Phys. Rev. Lett. 8 (1999) 362 9-3632 .

[9] FW. Starr, F Sciortino, H. E. Stanley, D ynamics of simulated water under pressure, Phys. Rev. Part E 60 (1999) 6757-6768 .

[10] W. Gotze, L. Sjogren, Relaxation processes in supercooled liquids, Rep. Prog. Phys. 5(5992 ) 2 41-376.

[11] C. A . A ngell, Relaxation in liquids, polymers and plastic crystals- strong/fragile patterns and problems, J. NonCryst. Sol. 131-13\$1991 ) 13-31.

[12] C. A . A ngell, Formation of glasses from liquids and biopolymers, Science 26 (1995) 19241935 .

[13] FH. Stillinger, A topographic view of supercooled liquids and glass formation, Science $267(1995)$ 1935-1939.

[14] H. Kanno, R. J. Speedy, C. A . A ngell, Supercooling of water to $-92{ }^{\circ} \mathrm{C}$ under pressure, Science $189(1975)$ 880 .

[15] O Mhima, H. E. Stanley, The relationship between liquid, supercooled and glassy water, Nature 396(1998) 32 9-335.

[16] E. Mlyer, New method for vitrifying water and other liquids by rapid cooling of their aerosols, J. A ppl. Phys. $56(1985) 663$.

[17] W. Kauzmann, The nature of the glassy state and the behavior of liquids at low temperatures, Chem. Rev. $\mathcal{B}$ (1948 ) 2 19-2 56 .

[18] G. A dam, J. H. Gibbs, @ the temperature dependence of cooperative relaxation properties in glass-forming liquids, J. Chem. Phys. (3196 5) 139-146.

[19] M Goldstein, Viscous liquids and the glass transition: a potential energy barrier picture, J. Chem. Phys. 51 (1969) $3728-3739$.

[2 $₫$ F H. Stillinger, T. A . Weber, Hidden structure in liquids, Phys. Rev. Part A 2(598 2) $978-989$.

[2 1 FH. Stillinger, T. A. Weber, Packing structures and transitions in liquids and solids, Science $22(5198$ 4) 98 3-989.

[2 1 P. G. D ebenedetti, FH. Stillinger, Supercooled liquids and the glass transition, Nature $410\left(\begin{array}{llll}2 & 0 & 0\end{array}\right) 1259-267$.

[2 $\$$ F Sciortino, P. Tartaglia, Harmonic dynamics in supercooled liquids: the case of water, Phys. Rev. Lett. 78 (1998) $2385-2388$.

[2 4 E. La Nave, A. Scala, FW. Starr, F Sciortino, H. E. Stanley, Instantaneous normal mode analysis of supercooled water, Phys. Rev. Lett. \$2400)046 0 5-460 8 . 


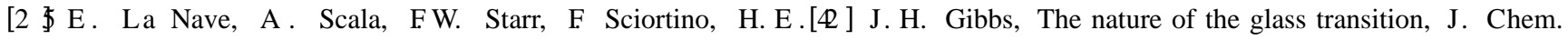
Stanley, D ynamics of super cooled water in configuration space, Phys. Rev. Part E $6\left(\begin{array}{lll}3 & 0 & 0\end{array}\right) 1036102$.

[2 $\$$ E. La Nave, H. E. Stanley, F Sciortino, Configuration space connectivity across the fragile- to- strong transition in silica, Phys. Rev. Lett. $\$ 2800) 2035501$.

[2 7 T. B. Schrøder, S. Sastry, J. D yre, S. C. Glotzer, Crossover to potential energy landscape dominated dynamics in a model glass- forming liquid, J. Chem. Phys. 1(20 00$) 0$ $9834-984$ ) . Phys. 2 \$1956) 185.

[4 ] S. Sastry, P. G. Debenedetti, F H. Stillinger, Signatures of distinct dynamical regimes in the energy landscape of a glass forming liquid, Nature 393 (1998 ) 554-557.

[4] ] F Sciortino, W. Kob, P. Tartaglia, Inherent structure entropy of supercooled liquids, Phys. Rev. Lett. 83 (1999) 32 14-32 17.

[45 ] S. Sastry, Oset temperature of slow dynamics in glass forming liquids, Phys. Chem. Comm. $1\left(\begin{array}{lll}2 & 0 & 0\end{array}\right) 0$

[2 \$ L. A ngelani, R. Di Leonardo, G. Ruocco, A. Scala, F [46] S. Sastry, Liquid limits: the glass transition and liquidSciortino, Saddles in the energy landscape probed by supercooled liquids, Phys. Rev. Lett. $8\left(\begin{array}{lll}2 & 0 & 0\end{array}\right)$ $5356-5359$.

[2 $\Phi$ C. J. Roberts, P. G. D ebenedetti, F H. Stillinger, E quation of state of the energy landscape of SPC/E water, J. Phys. Chem Part B $103(1999) 10258-10265$.

[30] K Broderix, K K Bhattacharya, A . Cavagna, A . Zippelius, I. Giardina, E nergy landscape of a LennardJones liquid: statistics of stationary points, Phys. Rev. Lett. 85200$) 05360-5363$.

[31] N. Giovambattista, FW. Starr, F Sciortino, H. E. Stanley, Transitions between inherent structures in water, Phys. Rev. Part E $6 \$ 200) 2041502$.

[32] N. Giovambattista, S. V. Buldyrev, FW. Stair, H. E. Stanley, Connection between A dam-Gibbs theory and spatially heterogeneous dynamics', Phys. Rev. Lett. 90 (2 00$) 3085506$.

[33] J. R. E rrington, P. G. D ebenedetti, S. Torquato, Cooperative origin of low- density domains in liquid water, Phys. Rev. Lett 8 \$2 00)22 15503.

[34] J. R. E rrington, P. G. D ebenedetti, Relationship between structural order and the anomalies of liquid water, Nature $409\left(\begin{array}{lll}2 & 0 & 0\end{array}\right) 1318-321$.

[35] A. Scala, FW. Starr, E. La Nave, F Sciortino, H. E. Stanley, Configurational entropy and diffusivity of supercooled water, Nature 40 6(2 00$) 0166-169$.

[36] F W. Starr, S. Sastry, E. La Nave, A. Scala, H. E. Stanley F Sciortino, Thermodynamic and structural aspects of the potential energy surface of simulated water, Phys. Rev. Part E $\left.6 \quad \begin{array}{llll}\hline & 0 & 0\end{array}\right) 1041201$.

[37] P. A . Netz, FW. Starr, H. E. Stanley, MC. Barbosa, Static and dynamic properties of stretched water, J. Chem. Phys. $115(200) 1344-348$.

[38] P. A . Netz, FW. Starr, MC. Barbosa, H. E. Stanley, Relation between structural and dynamical anomalies in supercooled water, Physica Part A $314\left(\begin{array}{lll}2 & 0 & 0\end{array}\right) 240-476$.

[39] F W. Starr, C. A. A ngell, H. E. Stanley, Prediction of entropy and dynamic properties of water below the homogeneous nucleation temperature', Physica Part A $322(200) 351-66$. gas spinodal boundaries of metastable liquids, Phys. Rev. Lett. 8(2000)0590-593.

[4] R. J. Speedy, The hard sphere glass transition, Mll. Phys. 95 (1998 ) 16 9-178.

[48] R. J. Speedy, Glass transition in hard sphere mixtures, J. Chem. Phys. 11(1999) 4559-4565.

[4] R. J. Speedy, Configurational entropy and diffusion in a hard disc fluid, J. Chem. Phys. 1 (27 0 0)190 $69-9074$

[50] S. Sastry, The relationship between fragility, configurational entropy and the potential energy landscape of glass forming liquids, Nature 40 9(2 00$)$ 116 4-16 7 .

[51] L. M Malrtinez, C. A . A ngell, A thermodynamic connection to the fragility of glassforming liquids, Nature 410 (2 00 ) $1663-667$.

[52] C. A. A ngell, S. Borick, Specific heáts $C_{v}, C_{\text {conf }}$ and energy landscapes of glassforming liquids, J. Non-Cryst. Sol. $307(200) 2393-406$.

[53] P. H. Poole, F Sciortino, U. Essmann, H. E. Stanley, Phase behavior of metastable water, Nature 360 (1992) $324-328$

[54] P. H. Poole, F Sciortino, U. E ssmann, H. E. Stanley, The spinodal of liquid water, Phys. Rev. Part E 1993 ) 3799-38 17.

[55] P. H. Poole, U. Essmann, F Sciortino, H. E. Stanley, Phase diagram for amorphous solid water, Phys. Rev. Part E 48 (1993) 460 5-46 10 .

[56] F Sciortino, P. H. Poole, U. E ssmann, H. E. Stanley, Line of compressibility maxima in the phase diagram of supercooled water, Phys. Rev. Part E 5\$1997) 72 7-737.

[57] S. Harrington, P. H. Poole, F Sciortino, H. E. Stanley, E quation of state of supercooled SPC/E water, J. Chem. Phys. $107(1997) 743-7450$

[58] S. Harrington, R. Zhang, P. H. Poole, F Sciortino, H. E. Stanley, Liquid-liquid phase transition: evidence from simulations, Phys. Rev. Lett. (8997) 2 409-2 412 .

[59] A. Scala, FW. Starr, E. La Nave, H. E. Stanley, F Sciortino, The free energy surface of supercooled water, Phys. Rev. Part E 6(200)08016-8020.

[4) ] C. A . A ngell, Water-II is a strong liquid, J. Phys. Chem. [6 d O Mhima, H. E. Stanley, D ecompression-induced 97 (1993) 6339-6 341 .

[41 ] V. Velikov, S. Borick, C. A. A ngell, The glass transition of water, based on hyperquenching experiments, Science $294(200) 12335-2338$. melting of ice IV and the liquid-liquid transition in water, Nature 392 (1998) 164-168.

[6 1] O Nhima, Liquid-liquid critical point in heavy water, Phys. Rev. Lett. \&(\$ 0 0)0334-336. 
[6 2] O Mhima, Y. Suzuki, Propagation of the polyamorphic transition of ice and the liquid-liquid critical point, Nature 419 (2 00 0)2599-6 03.

[6 3] P. H. Poole, F Sciortino, T. Grande, H. E. Stanley, C. A.[74] Yu. A. Qipov, B. V. Zheleznyi, N. F Bondarenko, The A ngell, E ffect of hydrogen bonds on the thermodynamic behavior of liquid water, Phys. Rev. Lett. 7(3 994 ) $1632-1635$

[64] C. J. Roberts, A. Z. Panagiotopoulos, P. G. D ebenedetti, PG, Liquid-liquid immiscibility in pure fluids: Polyamorphism in simulations of a network-forming fluid, Phys. Rev. Lett. 7(7996) $486-4389$.

[6 5] T. M Truskett, P. G. D ebenedetti, S. Sastry, S. Torquato, A single-bond approach to orientation-dependent interactions and its implications for liquid water, J. Chem. Phys. 111(1999) $2647-2656$.

[6 6 C. J. Roberts, P. G. D ebenedetti, Polyamorphism and density anomalies in network-forming fluids: Zerothand first-order approximations, J. Chem. Phys. 105 (1996) 658-672 .

[6 7] S. V. Buldyrev, G. Franzese, N. Giovambattista, G. Mllescio, MR. Sadr-Lahijany, A . Scala, A . Skibinsky, H. E . Stanley, Mdels for a liquid-liquid phase transition [Proceedings of the International Conference on Scattering Studies of Nascopic Scale Structure and D ynamics in Soft Malter ], Physica Part A $304\left(\begin{array}{lll}2 & 0 & 0\end{array}\right) 2$ $23-4$.

[6 \& A . Scala, MR. Sadr-Lahijany, N. Giovambattista, S. V. Buldyrev, H. E. Stanley, Water- like anomalies for coresoftened models of fluids: Two dimensional systems, Phys. Rev. Part E 6(3 0 0) 1041202 .

[69] G- Franzese, G. Mllescio, A . Skibinsky, S. V. Buldyrev, H. E. Stanley, Generic mechanism for generating a liquid-liquid phase transition, Nature 4) 9(2 0 0 $) 1692-695$.

[70] H. E. Stanley, J. Teixeira, Interpretation of the unusual behavior of $\mathrm{H}_{2}$ Oand D Qat low temperatures: Tests of a percolation model, J. Chem. Phys. 7\$1980) 340 434 2 .

[71] S. Sastry, P. G. D ebenedetti, F Sciortino, H. E. Stanley, Singularity-free interpretation of the thermodynamics of supercooled water, Phys. Rev. Part E 5(31996) $614-6154$

[72] L. P. N. Rebelo, P. G. D ebenedetti, S. Sastry, Singularityfree interpretation of the thermodynamics of supercooled water. II: Thermal and volumetric behavior, J. Chem. Phys. $109(1998) 626-633$.
[73] J. Hallett, The temperature dependence of the viscosity of supercooled water, Proc. Phys. Soc. $8(21963)$ $1046-1050$. shear viscosity of water supercooled to $-35^{\circ} \mathrm{C}$, Zh. Fiz. Khim. 51(1977) 12641265.

[75] K T. Gillen, D. C. D ouglass, MJ. R. Hoch, J. Chem. Phys. 57(1972) 5117.

[76] R. S. Smith, B. D. Kay, The existence of supercooled liquid water at $150 \mathrm{~K}$ Nature 398(1999) 78 8-791.

[77] R. Bohmer, K L. Ngai, C. A . A ngell, D. J. Plazek, Nonexponential relaxations in strong and fragile glass formers, J. Chem. Phys. 993$) \mathbb{Q} 01-\mathbb{Q} 09$.

[78] S. Sastry, Going strong or falling apart?, Nature 398 (1999) 467.

[79] I. Saika- Voivod, P. H. Poole, F Sciortino, Fragile- tostrong transition and polyamorphism in the energy landscape of liquid silica, Nature 42 (2 00$) 1514-517$.

[8 0] M Hemmati, C. T. Mynihan, C. A . A ngell, Interpretation of the molten $\mathrm{BeF}_{2}$ viscosity anomaly in terms of a high temperature density maximum, and other waterlike features, J. Chem. Phys. 1 (5 0 0)16663-66 71.

[8 1] C. A. A ngell, R. D. Bressel, M Hemmati, E. J. Sare, J. C. Tucker, Water and its anomalies in perspective; tetrahedral liquids with and without liquid-liquid phase transitions, Phys. Chem. Chem Phys. \& $(200) 0$ 1559-1566.

[8 2] W. Hage, A . Hallbrucker, E. Myer, G. P. Johari, Crystallization kinetics of water below $150 \mathrm{~K}$ J. Chem. Phys. $100(1994) 2$ 74-2 747.

[8 3] R. S. Smith, C. Huang, E. K L. Wong, B. D. Kay, D esorption and crystallization kinetics in nanoscale thin films of amorphous water ice, Surf. Sci. Lett. 36 7996) L13-L 18 .

[8 4 P. Jenniskens, D. F Blake, Crystallization of amorphous water ice in the solar system, A strophys. J. 4/31996) 1104

[8 5] K L. Ngai, J. H. Mgill, D. J. Plazek, How, diffusion and crystallization of supercooled liquids: revisited, J. Chem. Phys. $112(200) 01887-1892$.

[8 6 C. A . A ngell, Liquid fragility and the glass transition in water and aqueous solutions, Chem. Rev. 10(2 00$) 2$ $2627-2649$.

[8 7 G. P. Johari, D oes water need a neF, ? J. Chem. Phys. $116(200) 28067-8073$. 